\title{
Nuclear core activity reconstruction using heterogeneous instruments with data assimilation
}

\author{
Bertrand Bouriquet*, Jean-Philippe Argaud, Patrick Erhard, and Angélique Ponçot \\ Électricité de France, 1 avenue du Général de Gaulle, 92141 Clamart cedex, France
}

Received: 28 July 2015 / Received in final form: 8 October 2015 / Accepted: 20 November 2015

Published online: 18 December 2015

\begin{abstract}
Evaluating the neutronic state (neutron flux, power ...) of the whole nuclear core is a very important topic that has strong implication for nuclear core management and for security monitoring. The core state is evaluated using measurements and calculations. Usually, parts of the measurements are used, and only one kind of instrument is taken into account. However, the core state evaluation should be more accurate when more measurements are collected in the core. But using information from heterogeneous sources is at glance a difficult task. This difficulty can be overcome by Data Assimilation techniques. Such a method allows to combine in a coherent framework the information coming from numerical model and the one coming from various types of observations. Beyond the inner advantage to use heterogeneous instruments, this leads to obtaining a significant increase of the quality of neutronic global state reconstruction with respect to individual use of measures. In order to describe this approach, we introduce here the basic principles of data assimilation (focusing on BLUE, Best Unbiased Linear Estimation). Then we present the configuration of the method within the nuclear core problematic. Finally, we present the results obtained on nuclear measurements coming from various instruments.
\end{abstract}

\section{Introduction}

The knowledge of the neutronic state (neutron flux, power...) in the core is a fundamental point for the design, the safety and the production process of nuclear reactors. Due to the crucial role of this information, considerable work has been conducted for a long time to accurately estimate the neutronic spatial fields. Spatial distribution of power or activity in the whole core, or hottest point of the core, can be derived from such spatial fields. These information allow mainly to check that the nuclear reactor is working as expected in a very detailed manner, and that it will remain in the operating limits during production.

Two types of information can be used for the neutronic state evaluation.

Firstly, the physical core specifications, including the nuclear fuel description, make it possible to build a numerical simulation of the system. Taking into account neutronic, thermic and hydraulic spatial properties of the nuclear core, such well-known numerical models calculate in particular the reaction rates used for the physical analysis of the core state.

\footnotetext{
* e-mail: bertrand.bouriquet@edf.fr
}

Secondly, various measurements can be obtained from in-core or out-of-core detectors. Some detectors can measure neutron density, either locally or in spatially integrated areas, others can measure temperature of the incore water at some points. A lot of reliable measures come from periodical flux maps measured in each core reactor, at a periodicity of about one month. Then, all these measurements do not have the same type of physical relation with the neutronic activity, and also not the same accuracy. So it is not easy to take into account simultaneously all these heterogeneous measurements for the experimental evaluation of the neutronic state in the core.

A lot of these measures are local, in determined fuel assemblies, and do not give informations in un-instrumented areas of the core. The activity distribution over the whole core is traditionally obtained through an interpolation procedure, using the calculated fields as first guess (a proxy) of the "real" activity field corresponding to the measurements. In other words, the activity value in uninstrumented areas is calculated as the weighted average of the activity measures, using the calculated activity field to interpolate. The power is then obtained from activity through an observation operator, which depends only on core nominal physical specifications for the periodical flux map measurements. This interpolation procedure gives 
already good results, but some drawbacks remain in using only activity measures in a deterministic interpolation procedure.

Both physical core specifications and real measurements are subject to some uncertainties. Moreover, numerical assumptions, required to use the models, add some inaccuracy. All these uncertainties are not used explicitly in the interpolation procedure, but often used to qualify the a posteriori activity field obtained through the procedure. Moreover, the interpolation cannot take into account, for example, heterogeneous instruments, or observed discrepancy of some instruments.

Attempts have been made to overcome these limitations, mainly in two directions. Firstly, studies attempt to combine activity measurements and calculations through least-squares derived methods (for example in Ref. [1]), leading to the most probable activity (or power) field on the whole core. These methods allow to take into account heterogeneous measures, but are difficult to develop because of their extreme sensitivity to the weighting factor in the combination of measures and calculations. Secondly, explicit control of the error, in order to reduce its importance, has been tried through the development of adaptive methods to adjust coefficients in the calculation or the interpolation procedure.

Some of these difficulties can be solved by using data assimilation. This mathematical and numerical framework allows combining, in an optimal and consistent way, values obtained both from experimental measures and from a priori models, including information about their uncertainties. Commonly used in earth sciences as meteorology or oceanography [2], data assimilation has strong links with inverse problems or Bayesian estimation [3,4]. It is specifically tailored to solve such estimation problems through efficient yet powerful procedures such as Kalman filtering or variational assimilation [5,6]. Already introduced in nuclear field [7-10], it can be used both for field reconstruction or for parameter estimation in a unified formalism. In particular, in those papers are detailed effects of number and precision of measurements, as well as effect of instrument localization. Those methods are also used to improve nuclear data evaluation [11,12] as well as nuclear mass [13].

Data assimilation can treat information coming from any type of measure instruments, taking into account the way the measure is related with the objective field to be reconstructed, such as neutronic activity here. Data assimilation can further adapt itself to instrument configuration changes, and for example the removal or the failure of an instrument. Moreover, the method takes natively into account informations on instrumental or model uncertainties, introducing them a priori through the data assimilation procedure, and obtaining a posteriori the reduced uncertainties on the reconstruction solution.

In this paper, we introduce the data assimilation method and how it addresses physical field reconstruction. Then we make a detailed description of the various components that are used in data assimilation, and of the various types of instruments we can use to get in-core neutronic activity measurements. Then we present results with various instrument situations in nuclear core, obtained on a set of true nuclear cores.

\section{Data assimilation}

We briefly introduce the useful data assimilation key points, to understand their use as applied here. But data assimilation is a wider domain, and these techniques are for example the keys of nowadays meteorological operational forecast. It is through advanced data assimilation methods that long-term forecasting of the weather has been drastically improved in the last 30 years. Forecasting is based on all the available data, such as ground and satellite measurements, as well as sophisticated numerical models. Some interesting information on these approaches can be found in the following basic references $[2,5,6]$.

The ultimate goal of data assimilation methods is to be able to provide a best estimate of the inaccessible "true" value of the system state (denoted $\mathbf{x}^{t}$, with the $t$ index standing for "true"). The basic idea of data assimilation is to put together information coming from an a priori state of the system (usually called the "background" and denoted $\mathbf{x}^{b}$ ), and information coming from measurements (denoted as $\mathbf{y}$ ). The result of data assimilation is called the analysed state $\mathbf{x}^{a}$ (or the "analysis"), and it is an estimation of the true state $\mathbf{x}^{t}$ we want to find. Details on the method can be found in references [4] or [5].

Mathematical relations between all these states need to be defined. As the mathematical spaces of the background and of the observations are not necessarily the same, a bridge between them has to be built. This bridge is called the observation operator $H$, with its linearisation $\mathbf{H}$, that transforms values from the space of the background state to the space of observations. The reciprocal operator is known as the adjoint of $H$. In the linear case, the adjoint operator is the transpose $\mathbf{H}^{T}$ of $\mathbf{H}$.

Two additional pieces of information are needed. The first one is the relationships between observation errors in all the measured points. They are described by the covariance matrix $\mathbf{R}$ of observation errors $\varepsilon_{0}$, defined by $\varepsilon_{0}=\mathbf{y}-H\left(\mathbf{x}^{t}\right)$. It is assumed that the errors are unbiased, so that $E\left[\varepsilon_{0}\right]=0$, where $E$ is the mathematical expectation. $\mathbf{R}$ can be obtained from the known errors on the unbiased measurements. The second one is similar and describes the relationships between background errors. They are described by the covariance matrix $\mathbf{B}$ of background errors $\varepsilon_{b}$, defined by $\varepsilon_{b}=\mathbf{x}^{b}-\mathbf{x}^{t}$. This represents the a priori error, assuming it to be also unbiased. There are many ways to obtain this a priori and background error matrices. However, in practice, they are commonly built from the output of a model with an evaluation of its accuracy, and/or the result of expert knowledge.

It can be proved, within this framework, that the analysis $\mathbf{x}^{a}$ is the Best Linear Unbiased Estimator (BLUE), and is given by the following formula:

$$
x^{a}=x^{b}+K\left(y-H x^{b}\right),
$$

where $\mathbf{K}$ is the gain matrix [5]:

$$
K=B H^{T}\left(H B H^{T}+R\right)^{-1} .
$$

Moreover, we can get the analysis error covariance matrix $\mathbf{A}$, characterising the analysis errors $\varepsilon_{a}=\mathbf{x}^{a}-\mathbf{x}^{t}$. 
This matrix can be expressed from $\mathbf{K}$ as:

$$
A=(I-K H) B,
$$

where $\mathbf{I}$ is the identity matrix.

The detailed demonstrations of those formulas can be found in particular in the reference [5]. We note that, in the case of Gaussian distribution probabilities for the variables, solving equation (1) is equivalent to minimising the following function $J(\mathbf{x}), \mathbf{x}^{a}$ being the optimal solution:

$$
\begin{aligned}
J(x)= & \left(x-x^{b}\right)^{T} B^{-1}\left(x-x^{b}\right) \\
& +(y-H x)^{T} R^{-1}(y-H x) .
\end{aligned}
$$

We can make some enlightening comments concerning this equation (4), and more generally on the data assimilation methodology. If we do extreme assumptions on model and measurements, we notice that these cases are covered by minimising $J$. Firstly, assuming that the model is completely wrong, then the covariance matrix $\mathbf{B}$ is $\infty$ (or equivalently $\mathbf{B}^{-1}$ is 0 ). The minimum of $J$ is then given by $\mathbf{x}^{a}=\mathbf{H}^{-1} \mathbf{y}$ (denoting by $\mathbf{H}^{-1}$ the inverse of $\mathbf{H}$ in the least square sense). It corresponds directly to information given only by measurements in order reconstruct the physical field. Secondly, on the opposite side, the assumption that measurements are useless implies that $\mathbf{R}$ is $\infty$. The minimum of $J$ is then evident: $\mathbf{x}^{a}=\mathbf{x}^{b}$ and the best estimate of the physical field is then the calculated one. Thus, such an approach covers the whole range of assumptions we can have with respect to models and measurements.

\section{Data assimilation method parameters}

The framework of the study is the standard configuration of a 1300 electrical MW Pressurized Water Reactor (PWR1300) nuclear core. Our goal is to reconstruct the neutronic fields, such as the activity, in the active part of the nuclear core. For that purpose, we use data assimilation. To implement such methodology, we need both simulation codes and measures. For the simulation code, we use standard EDF calculation code COCCINELLE for nuclear core simulation, in a typical configuration (see Ref. [14] for a general overview). The results are built on a set of 20 experimental neutronic flux maps measured on various PWR1300 nuclear cores. Such measurements are done periodically (about each month) on each nuclear core. These different measurement situations are chosen for their representativeness, in order that statistical results cover a wide range of situations and can have some sort of predictability property.

\subsection{The background and the measurements}

A standard PWR1300 nuclear core has 193 fuel assemblies within. For the calculation, those assemblies are each considered as homogeneous, and are divided in 38 vertical levels. Thus, the state field $\mathbf{x}$ can be represented as a vector of size $193 \times 38=7334$.
The background is built upon neutronic diffusion calculation from operational COCCINELLE code routinely used at EDF. This code produces the fields for neutron flux, power and temperature in the context of real cores.

The measurements come from instruments that can be located on horizontal 2D maps of the core. There are three types of instrument that are usually used to monitor the nuclear power core:

- Mobile Fission Chambers (MFC), which measure neutrons inside the active part of the nuclear core, and for which the locations are presented on Figure 1;

- Thermocouples (TC), which are above the active nuclear core, for which the locations are presented on Figure 2;

- fixed ex-core detector locations.

The data coming from the ex-core detectors are continuous in time and are very efficient for security purpose, which is their main goal. Their purpose is to continuously monitor the core, but not to measure accurately the neutronic activity at each fine flux map. So, their measures are too crude for being interesting on a fine reconstruction of the inner core activity map. Thus, we choose here to not take into account information coming from those ex-core detectors.

All these types of instrumentation (MFC, TC, ex-core) can be found on any power plants. For the purpose of this study, we add artificially an extra type of detector, described as idealized Low Granularity MFC (named here LMFC). The measurement attributed to the LMFC are built artificially from the information given by the MFC. Thus they are replacing the MFC on the given LMFC locations. The evaluation of LMFC response is calculated from the MFC measured neutron flux, assuming a different physic process, and a lower granularity. The lower granularity assumption done on the LMFC induces a partial integration of the results of the MFC over a given area. Of course, the physical process involved to make a measurement being different, the resolution of LMFC will be different from the one from MFC. We take 16 of those instruments. They are located in various area of the core, replacing MFC, to try to make a representative array of measurement as shown on Figure 3.

The main characteristics of the instruments, as their number in the core, the number of considered vertical levels, and the size of the part of the observation vector $\mathbf{y}$ associated with the particular instrument type, are reported in Table 1 . The size of the final observation vector is given by summing the size of all the individual $\mathbf{y}$ vector of the instruments used.

\subsection{The observation operator $\mathrm{H}$}

As the output of the neutronic code COCCINELLE provides results which are equivalent to measurements, the observation operator $\mathbf{H}$ is mainly a selection operator, that picks up the chosen information for an instrument among all the code outputs. A normalisation procedure is added for the measurements that have no absolute value.

In details, the $\mathbf{H}$ observation operator can be built independently for each instrument. Each observation 


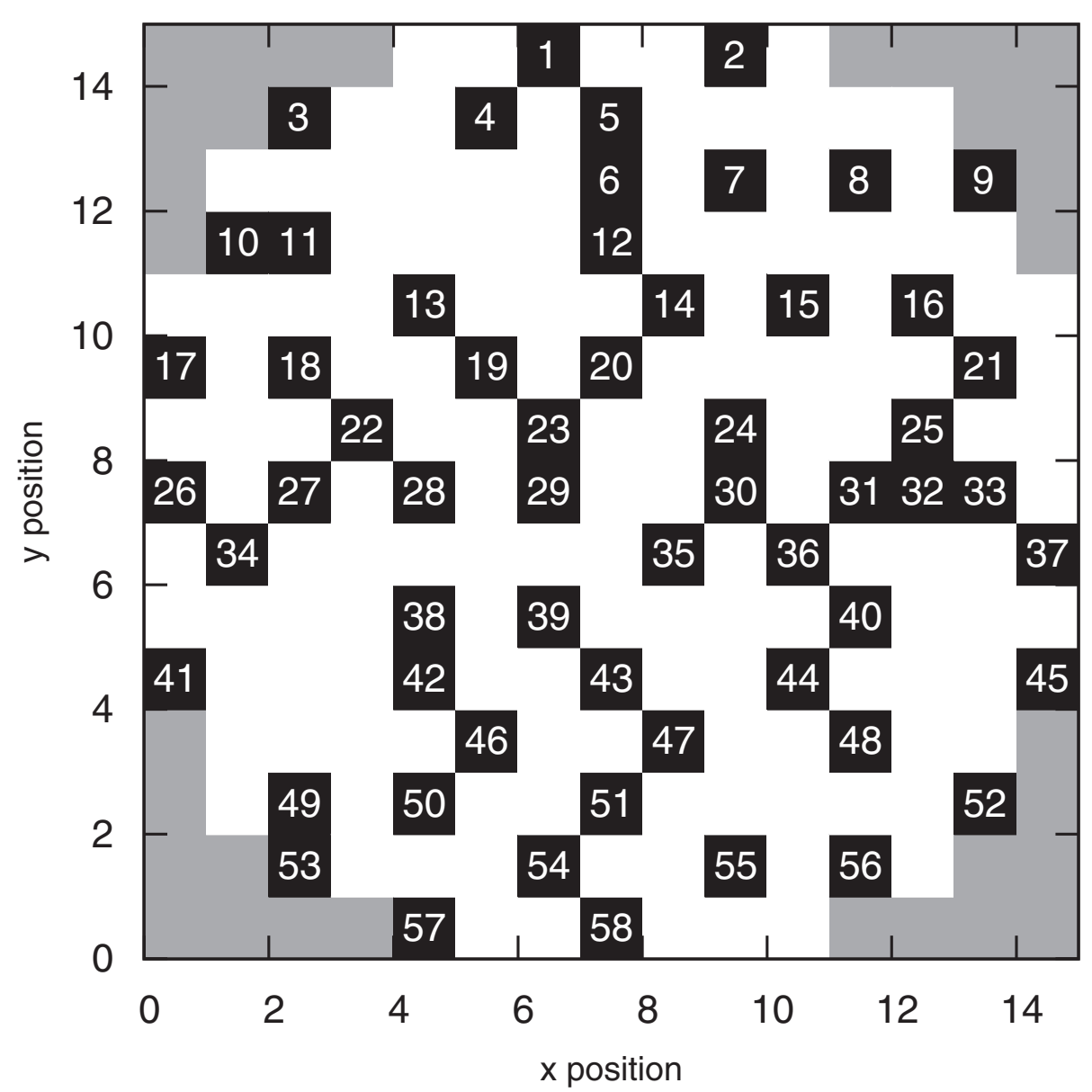

Fig. 1. The Mobile Fission Chambers (MFC) instruments within the nuclear core are localised in assemblies in black, in a horizontal slice of the core. The assemblies without instrument are marked in white, and the reflector is in grey.

operator is then basically a selection matrix, that chooses in the model space a cell that is involved in a measurement in the observation space. In addition, a weight, according to the size of the cell, is affected to the selection. As some experimental data are normalised, this selection matrix is multiplied by a normalisation matrix that represents the effect of the cross normalisation of the data. This observation matrix is a $\left(7334 \times \sum d_{i}\right)$ matrix, where $d_{i}$ is the size of the part of the observation vector $\mathbf{y}$ for each instrument involved in assimilation, as reported in Table 1.

So there is one individual $\mathbf{H}$ matrix observation operator by instrument type. The complete $\mathbf{H}$ matrix observation operator is the concatenation, as a blocdiagonal matrix, of all the individual matrix for each instrument.

\subsection{The background error covariance matrix $B$}

The $\mathbf{B}$ matrix represents the covariance between the spatialised errors for the background. The $\mathbf{B}$ matrix is estimated as the double-product of a correlation matrix $\mathbf{C}$ by a diagonal scaling matrix containing standard deviation, to set variances.

The correlation $\mathbf{C}$ matrix is built using a positive function that defines the correlations between instruments with respect to a pseudo-distance in model space. Positive functions allow, through the Bochner theorem, to build symmetric defined positive matrix when they are used as matrix generator (for theoretical insight, see reference documents [15] and [16]). Second Order Auto-Regressive (SOAR) function is used here. In such a function, the amount of correlation depends from the euclidean distance between spatial points in the core. The radial and vertical correlation lengths (denoted $L_{r}$ and $L_{z}$ respectively, associated to the radial $r$ coordinate and the vertical $z$ coordinate) have different values, which means we are dealing with a global pseudo euclidean distance. The used function can be expressed as follows:

$$
C(r, z)=\left(1+\frac{r}{L_{r}}\right)\left(1+\frac{|z|}{L_{z}}\right) \exp \left(-\frac{r}{L_{r}}-\frac{|z|}{L_{z}}\right)
$$

The matrix $\mathbf{C}$ obtained from the above equation (5) is a correlation one. It can be multiplied (on left and right) by a suitable diagonal standard deviation matrix, to get covariance matrix. If the error variance is spatially constant, there is only one coefficient to multiply $\mathbf{C}$. This coefficient is obtained here by a statistical study of difference between the model and the measurements in 


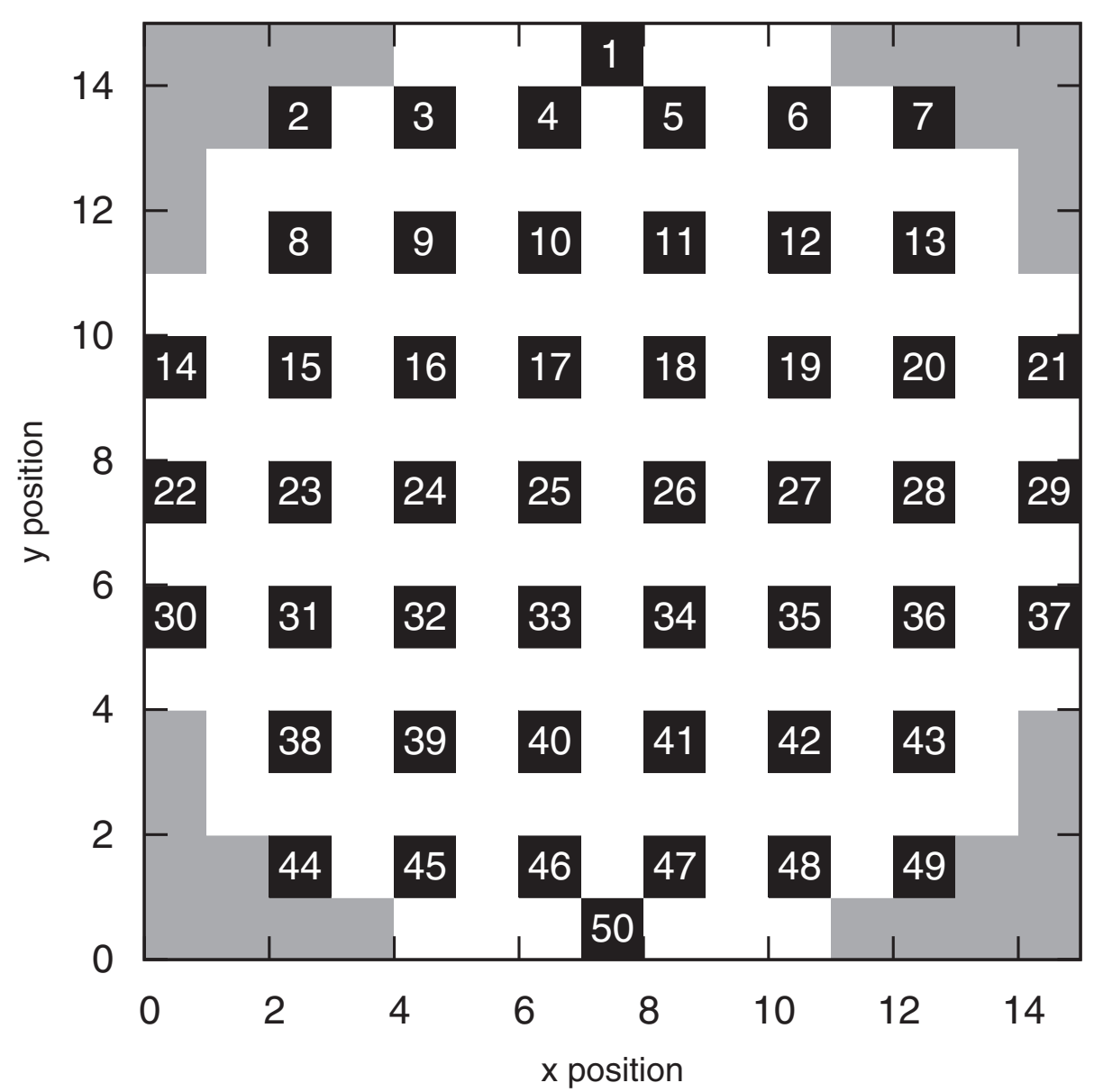

Fig. 2. The Thermocouples (TC) instruments within the nuclear core are localised above assemblies in black, in a horizontal slice of the core. The assemblies without instrument are marked in white, and the reflector is in grey.

real cases. In real cases, this value is set around a few percent.

Globally speaking, the covariance matrix is fully defined by the parameters $L_{r}$ and $L_{z}$ that are related to the mean diffusion length of neutrons in the assemblies.

The size of the background error covariance matrix $\mathbf{B}$ is related to the size of model space, so it is $(7334 \times 7334)$ here.

\subsection{The observation error covariance matrix $R$}

The observation error covariance matrix $\mathbf{R}$ is approximated by a simple diagonal matrix. It means we assume that no significant correlation exists between the measurement errors of all the instruments. A usual modelling consists in taking the diagonal values as a percentage of the observation values. This can be expressed as:

$$
R_{j j}=\left(\alpha y_{j}\right)^{2}, \quad \forall j
$$

The $\alpha$ parameter is fixed according to the accuracy of the measurements and the representative error associated to the instruments. It is the same for all the diagonal coefficients related with one instrument. Its value only depends on the type of instrument we are dealing with. The $\alpha$ value can be determined by both statistical method and expert opinion about the measurement quality. In the present paper, we will use arbitrary value for the $\alpha$.

The size of the $\mathbf{R}$ matrix is related to the size of the observation space, so it is $\left(\sum_{i} d_{i} \times \sum_{i} d_{i}\right)$ where $d_{i}$ is the size of the observation vector of each instrument $i$ involved in assimilation, as reported in Table 1.

\section{Results on data assimilation using only one type of instrument}

The first results are showing the quality of the reconstruction as a function of the various types of instruments that are taken into account for reconstructing the activity of the core.

The experimental data are a set of measurements on the 38 levels of all the instrument locations inside of the core. Thus, to evaluate the quality of the reconstruction of the physical fields with one type of instrument, we look for the misfit $\left(\mathbf{y}-\mathbf{H} \mathbf{x}^{a}\right)$ at measurement locations (by other instruments) that are not involved in the assimilation process. The number of locations, where there is a 


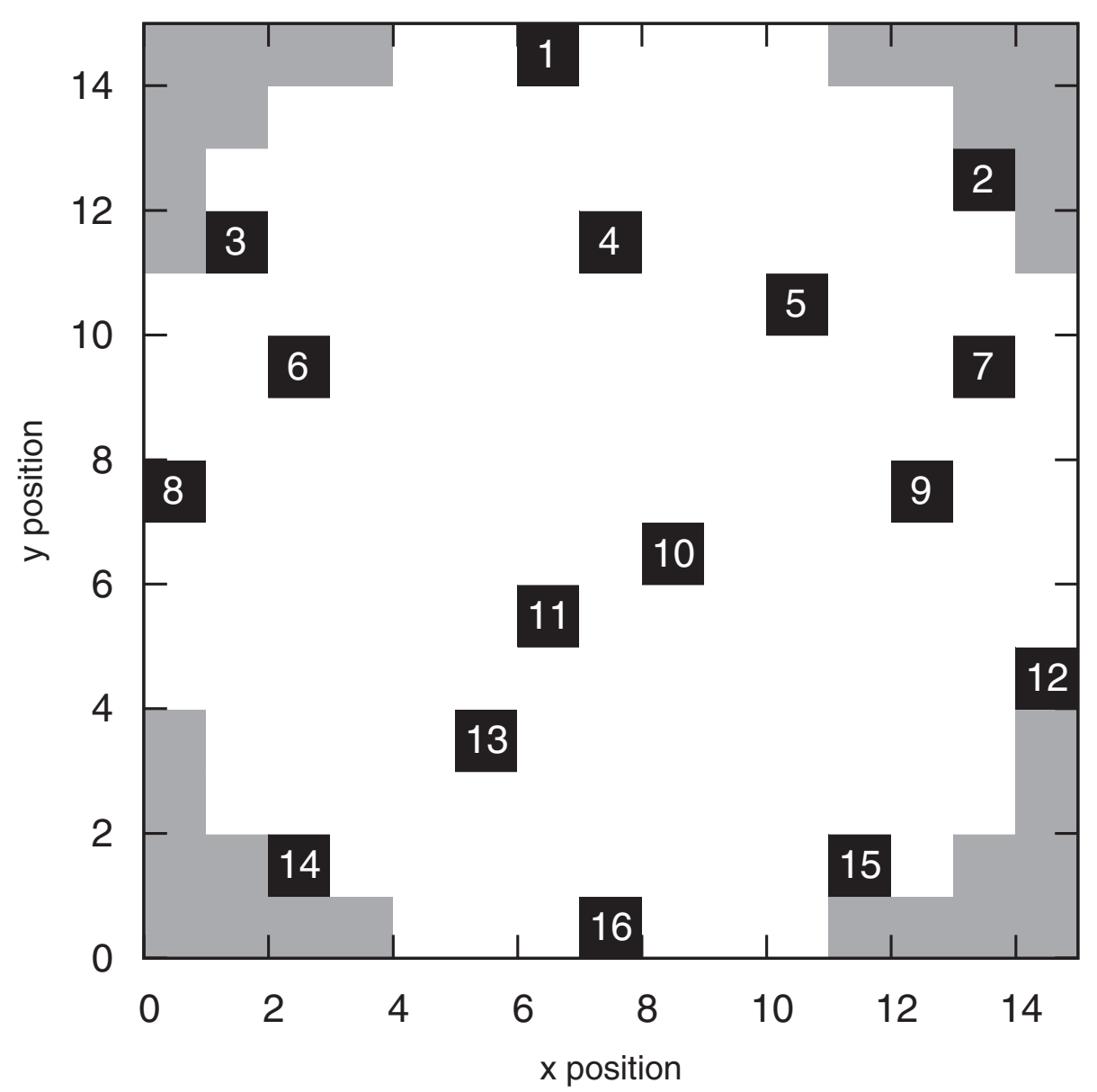

Fig. 3. The idealized Low Granularity MFC (LMFC) instruments within the nuclear core are localised in assemblies in black, in a horizontal slice of the core. The assemblies without instrument are marked in white, and the reflector is in grey.

measurement and that is not involved in data assimilation procedure (so where the misfit $\left(\mathbf{y}-\mathbf{H} \mathbf{x}^{a}\right)$ is calculated) is synthesised in Table 2 , as well as the accuracy associated to each instrument through the $\alpha$ parameter of equation (6).

Thermocouples being a fully integral measurement outside of the active core, we can do the misfit $\left(\mathbf{y}-\mathbf{H} \mathbf{x}^{a}\right)$ evaluation of the reconstruction in all the locations of $\mathrm{MFC} / \mathrm{LMFC}$.

For each data assimilation procedure associated with an instrument, we calculate the Root Mean Square (RMS, which is the norm) of the misfit $\left(\mathbf{y}-\mathbf{H} \mathbf{x}^{a}\right)$ on all the misfit calculation locations. To synthesize the value for one set of measurement, we take the mean value of the misfit
$\left(\mathbf{y}-\mathbf{H} \mathbf{x}^{a}\right)$ on each of the 38 levels of the core, which leads to a horizontally averaged value of the misfit $\left(\mathbf{y}-\mathbf{H} \mathbf{x}^{a}\right)$. For the sake of more general behaviour, we take 20 sets of flux map measurements, with various settings and ageing of PWR1300 nuclear cores. Then we proceed to the calculation of the mean value on all those sets of measurement.

Globally all the results present strong misfit $\left(\mathbf{y}-\mathbf{H} \mathbf{x}^{a}\right)$ on the upper and lower levels, which is a known effect mainly due to the axial reflector modelling. In addition, the central part of the reactive core in the nuclear plant is also the region where the neutron flux is the most intense, so the hot spot of the activity field in the core is expected to be in this region. Thus, the next plots are restricted to the centre of the core.

Table 1. Main characteristics of the instruments used for data assimilation. These characteristics remain the same, either in mono-instrumented cases or in multi-instrumented ones.

\begin{tabular}{lllr}
\hline $\begin{array}{l}\text { Instrument } \\
\text { type }\end{array}$ & $\begin{array}{l}\text { Locations } \\
\text { number }\end{array}$ & $\begin{array}{l}\text { Vertical } \\
\text { levels }\end{array}$ & $\begin{array}{l}\text { Size of the } \mathbf{y} \\
\text { vector part }\end{array}$ \\
\hline MFC & 42 & 38 & 1596 \\
LMFC & 16 & 8 & 128 \\
TC & 50 & 1 & 50 \\
\hline
\end{tabular}


Table 2. Number of misfit calculation locations used for each instrument type considered individually for data assimilation, and arbitrary accuracy assumed in the present studies for each type of instrument.

\begin{tabular}{lll}
\hline $\begin{array}{l}\text { Instrument } \\
\text { type }\end{array}$ & $\begin{array}{l}\text { Number of misfit } \\
\text { calculations locations }\end{array}$ & $\begin{array}{l}\alpha \text { value } \\
(\%)\end{array}$ \\
\hline MFC & 16 & 1 \\
LMFC & 42 & 2 \\
TC & 58 & 3 \\
\hline
\end{tabular}

Figure 4 shows the axial misfit measured by the standard RMS of the difference between analysis and measurements, in arbitrary units, on all the data assimilation unused locations for the various types of instrument studied. The oscillating behaviours, that are barely noticeable on all the curves, come from the different materials that are within a core level, and mainly the steel grids that maintain assemblies. Some levels contain a mechanical structure of the core, thus these are more neutron absorption.

We noticed, as expected, that the reconstruction coming from the thermocouples (TC) is the closest to the background, due to their integral measurement property and their lower accuracy. Moreover, an improvement of the accuracy does not improve dramatically the quality of the core state evaluation, mainly due to the integral measurement property.

The Low Granularity MFC (LMFC) are showing a good reconstruction of the physical field in the whole core, despite the not so good accuracy and the limited number of measurements. The increase of the misfit from $\left(\mathbf{y}-\mathbf{H} \mathbf{x}^{b}\right)$ to $\left(\mathbf{y}-\mathbf{H x}^{a}\right)$ for the lower part of the core is easily explainable by the chosen locations of LMFC that are in core. This part, near the border of the core, does not get enough measurements to be very accurately reconstructed.

The reconstruction using only MFC is, also as expected from accuracy values, the best one. The data assimilation procedure leads to half the misfit $\left(\mathbf{y}-\mathbf{H x}^{b}\right)$ observed when only using the model.

From results coming from MFC and LMFC, we notice that, within the hypothesis and the chosen modelling of the integration, TC measurement permits only a crude evaluation of the core state.

\section{Results on data assimilation with heterogeneous instruments}

This section describes results using different instruments together in the data assimilation procedure.

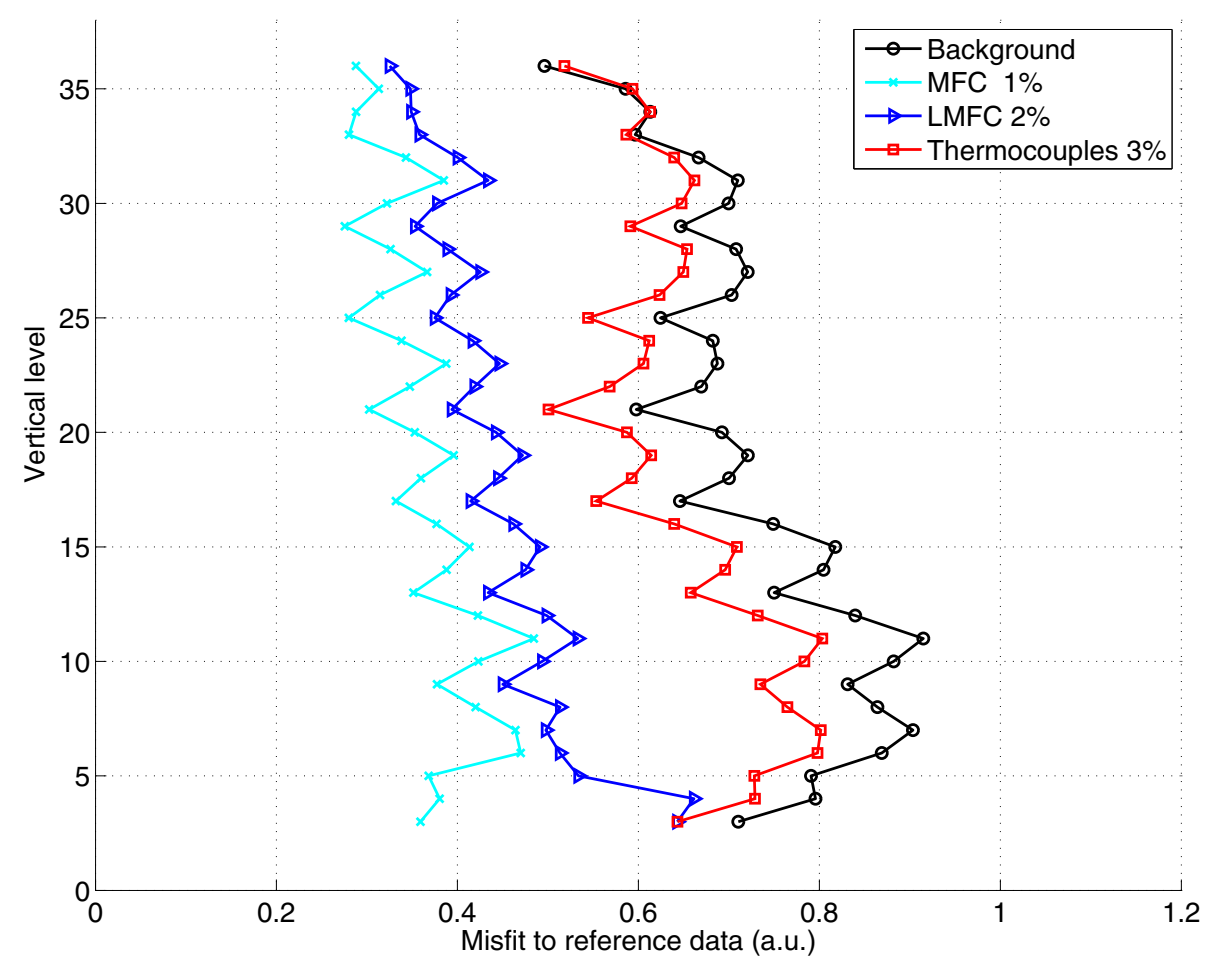

Fig. 4. Vertical misfit for various kind of intruments, measured by the RMS of the differences between the analysis and the measurements at unused locations (see text for details). 


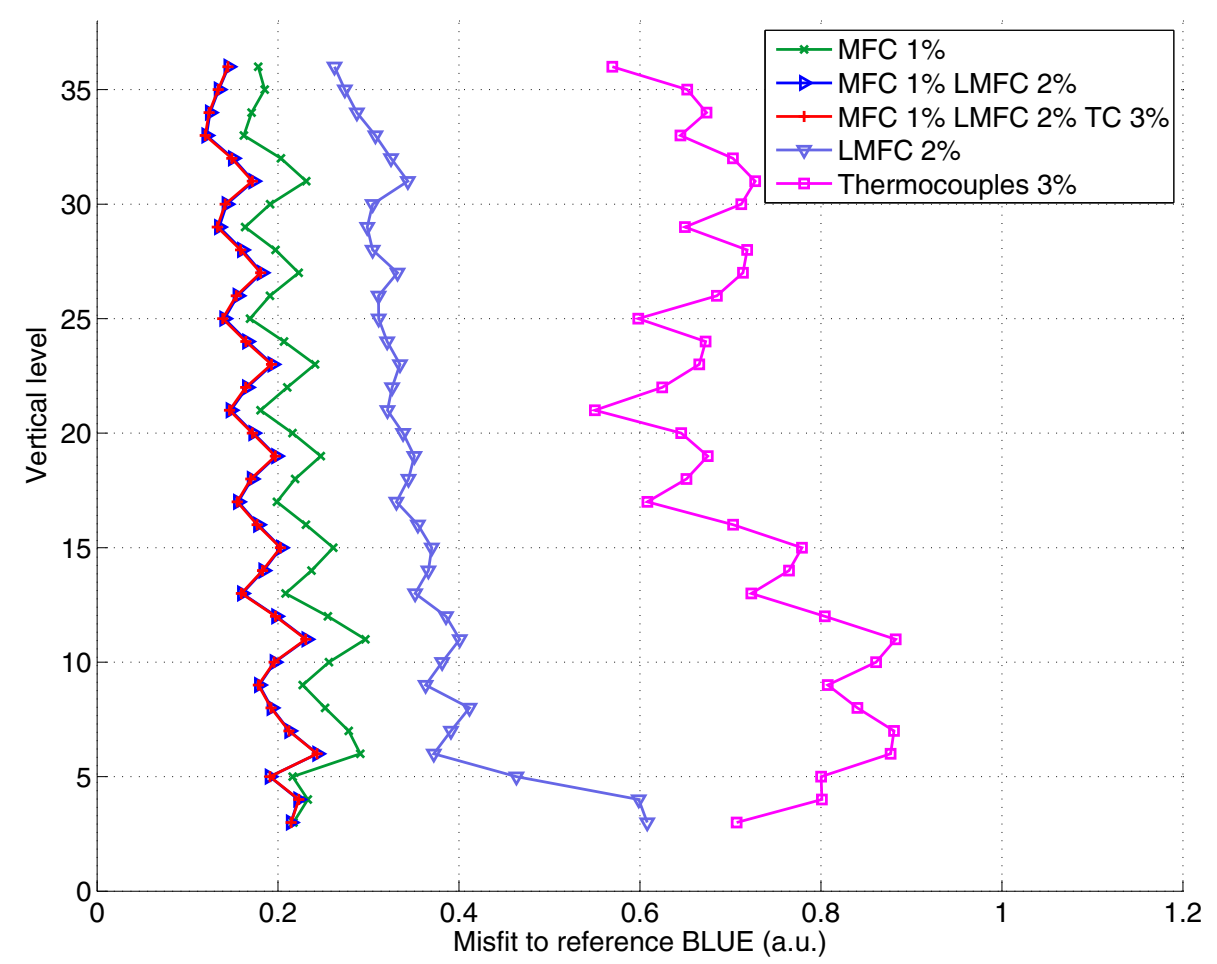

Fig. 5. Axial misfit for various kind of instrumental configurations, measured by the RMS of the difference between the analysis and the reference calculation.

In this case, we cannot take as reference the measures points taken apart, as when we are studying individual instruments as presented in Section 4. Thus, we choose to make an analysis with data assimilation using all the available measures in the core, on the 58 locations, and using a $1 \%$ accuracy. Then we evaluate the misfit $\left(\mathbf{y}-\mathbf{H x}^{a}\right)$ with respect to this reference calculation in all the assemblies where no MFC are present, which means 135 locations (193 total assembly minus 58 instrumented locations). The calculated values at those instrumented locations allow to benchmark the quality of the reconstruction. On this misfit $\left(\mathbf{y}-\mathbf{H x}^{a}\right)$, we calculate the RMS per horizontal level as previously, and take the average over some 20 selected flux map measurements. The results are presented on Figure 5.

Figure 5 presents RMS per horizontal level of the misfit calculated for various instruments taken alone or in conjunction. We notice that the results have the same behaviour as the one we got in Figure 4. This means that the reference we chose to evaluate the quality of data assimilation using several instrument types can be considered as reliable.

Looking at the successive addition of instrument, we notice that addition of the LMFC has an important effect, the MFC + LMFC configuration presenting an improvement with respect to the configuration with only MFC. However, adding thermocouple to this configuration is not really helpful, and the improvement is not really noticeable on Figure 5.

These results highlight a number of important points on data assimilation methodology. On one hand, when only very few measurements are available, they are very helpful and allow a fairly good improvement of reconstructed physical field. On the other hand, when a lot of measurements are available, adding a few more, or with a lower accuracy as thermocouples, does not change dramatically the result of field reconstruction by data assimilation. On overall, this shows that data assimilation technique is doing the best use of experimental information provided to the procedure. Those results are comforting the ones found on the robustness of the evaluation of the nuclear core by data assimilation, when only MFC is used as presented in reference [9]. Moreover, as expected in data assimilation technique, the use of heterogeneous instruments is integrated within the method.

\section{Conclusion}

The use of data assimilation has already been proved to be efficient to reconstruct fields in several domains, and recently in neutronic activity field reconstruction for nuclear core. The present paper demonstrates that, within the data assimilation framework, information coming from heterogeneous sources can be used without making any adjustment to the method.

Looking at the various types of instruments we have (MFC, LMFC and TC), we notice that the influence they have on reconstruction depends on three parameters:

- the granularity of each type of instrument, that is the density of instrument, their integral measurement property and their repartition all over the core;

- the accuracy of each instrument, possibly with respect to the accuracy of the others; 
- and the global instrumentation configuration, that is the complex repartition of all instruments.

Those conclusions arise from the comparison of the various instruments, using them individually or together, in various instrumental configurations.

Data assimilation gives a very efficient and adaptable framework in order to take the best from both experimental data and model. Moreover, this can be done without heterogeneities constraints between instruments. This technique is used here in a very elementary way, but it opens the door to many developments, for example in systematic data analysis, models comparison, in dynamic modelling, etc. for nuclear reactors.

\section{References}

1. H. Ezure, Estimation of most probable power distribution in BWRs by least squares method using in-core measurements, J. Nucl. Sci. Technol. 25, 731 (1988)

2. E. Kalnay, Atmospheric modeling, data assimilation and predictability (Cambridge University Press, 2003)

3. S.M. Uppala et al., The ERA-40 re-analysis, Q. J. R. Meteorol. Soc. 131, 2961 (2005)

4. A. Tarantola, Inverse problem: theory methods for data fitting and parameter estimation (Elsevier, 1987)

5. F. Bouttier, P. Courtier, Data assimilation concepts and methods, Meteorological training course lecture series, ECMWF, 1999

6. O. Talagrand, Assimilation of observations, an introduction, J. Meteorol. Soc. Jpn 75, 191 (1997)

7. S. Massart, S. Buis, P. Erhard, G. Gacon, Use of 3DVAR and Kalman filter approaches for neutronic state and parameter estimation in nuclear reactors, Nucl. Sci. Eng. 155, 409 (2007)
8. B. Bouriquet, J.-P. Argaud, P. Erhard, S. Massart, A. Ponçot, S. Ricci, O. Thual, Differential influence of instruments in nuclear core activity evaluation by data assimilation, Nucl. Instrum. Methods Phys. Res. A 626-627, 97 (2011)

9. B. Bouriquet, J.-P. Argaud, P. Erhard, S. Massart, A. Ponçot, S. Ricci, O. Thual, Robustness of nuclear core activity reconstruction by data assimilation, Nucl. Instrum. Methods Phys. Res. A 629, 282 (2011)

10. B. Bouriquet, J.-P. Argaud, R. Cugnart, Optimal design of measurement network for neautronic activity field reconstruction by data assimilation, Nucl. Instrum. Methods Phys. Res. A 664, 117 (2012)

11. P. Blaise, D. Bernard, C. de Saint Jean, N. dos Santos, P. Leconte, A. Santamarina, J. Tommasi, J.-F. Vidal, The uncertainty quantification for complex neutronics code packages: issues on modern nuclear data assimilation and transposition to future nuclear systems, in ASME $2011 \mathrm{PVP}$ MF Symposium on risk-informed monitoring, modeling, and operation of aging systems Baltimore, Maryland, USA, July 17-22, 2011 (2011)

12. D.W. Muir, The contribution of individual correlated parameters to the uncertainty of integral quantities, Nucl. Instrum. Methods Phys. Res. A 644, 55 (2011)

13. B. Bouriquet, J.-P. Argaud, Best linear unbiased estimation of the nuclear masses, Ann. Nucl. Energy 38, 1863 (2011)

14. A. Santamarina, C. Collignon, C. Garat, French calculation schemes for light water reactor analysis, in PHYSOR 2004 The Physics of Fuel Cycles and Advanced Nuclear Systems: Global Developments Chicago, Illinois, April 25-29, 2004, on CD-ROM, American Nuclear Society, Lagrange Park, IL, 2004 (2004)

15. G. Matheron, La théorie des variables régionalisées et ses applications, Cahiers du Centre de Morphologie Mathématique de l'ENSMP, Fontainebleau, Fascicule 5, 1970

16. D. Marcotte, Géologie et géostatistique minières (lecture notes), 2008

Cite this article as: Bertrand Bouriquet, Jean-Philippe Argaud, Patrick Erhard, Angélique Ponçot, Nuclear core activity reconstruction using heterogeneous instruments with data assimilation, EPJ Nuclear Sci. Technol. 1, 18 (2015) 